\title{
ОЦЕНКА ЭФФЕКТИВНОСТИ МЕРОПРИЯТИЙ ИНФОРМАЦИОННОЙ БЕЗОПАСНОСТИ НА ЗАЩИЩЕННЫХ ЭКОНОМИЧЕСКИХ СИСТЕМАХ С ПРИМЕНЕНИЕМ ИСКУССТВЕННЫХ НЕЙРОННЫХ СЕТЕЙ
}

\author{
(c) 2019 Скляров Алексей Викторович \\ кандидат технических наук, доцент \\ Ростовский государственный экономический университет (РИНХ), Россия, Ростов-на-Дону \\ E-mail: SAV0701@mail.ru
}

(c) 2019 Тищенко Евгений Николаевич

доктор экономических наук, профессор

Ростовский государственный экономический университет (РИНХ), Россия, Ростов-на-Дону

\section{(c) 2019 Ефимова Елена Владимировна}

кандидат экономических наук, доцент

Ростовский государственный экономический университет (РИНХ), Россия, Ростов-на-Дону

\section{(c) 2019 Жилина Елена Викторовна}

кандидат экономических наук, доцент

Ростовский государственный экономический университет (РИНХ), Россия, Ростов-на-Дону

Предложен подход к решению задачи оценки эффективности мероприятий информационной безопасности на защищенных экономических системах, в котором для обработки экспертных оценок применена искусственная нейронная сеть, что позволяет существенно повысить объективность и точность оценивания и, как следствие, его качество.

Ключевые слова: информационная безопасность, эффективность, искусственные нейронные сети.

В современных условиях обеспечение информационной безопасности защищенных экономических систем происходит при воздействии на них множества факторов, имеющих, как правило, стохастический характер способных существенно снизить эффективность и даже полностью скомпрометировать защитные мероприятия. При этом, в ходе организации защиты критически важных для собственников информационных ресурсов, компании руководствуются требованиями российских и международных стандартов, регламентирующих деятельность в этой области. Однако, указанные нормативные документы практически не содержат конкретных методик и носят скорей декларативный или рекомендательный характер. По мнению авторов, такому положению дел есть ряд причин:

- при разработке защитных мероприятий не учитывается экономическое содержание и вероятностный характер событий и явлений, возникающих в процессе реализации этих мероприятий;

- отставание нормативной базы от потреб- ностей практики вследствие исключительно высоких темпов развития информационных технологий. При этом, в сфере информационной безопасности подобное отставание оказывается особенно критичным;

- несовершенство нормативного обеспечения защиты информации, которое проявляется в фактическом отсутствии системы показателей защищенности и критериев безопасности объектов информатизации.

В результате отсутствует возможность оценки эффективности планируемых и уже реализованные защитных мер, под которой понимают степень соответствия результатов защиты информации поставленной цели [1].

Объективным видом оценки эффективности систем защиты информации (СЗИ) является функциональное тестирование, предназначенное для проверки фактической работоспособности реализованных механизмов безопасности и их соответствия предъявленным требованиям, а также обеспечивающее получение статистических данных. В методическом плане опреде- 
ление эффективности СЗИ заключается в выработке суждения относительно пригодности способа действий персонала или приспособленности технических средств к достижению цели защиты информации на основе измерения соответствующих показателей, например, при функциональном тестировании. Такие данные, получаются экспериментально, посредством математического моделирования или путем экспертных оценок. При этом в соответствии с современной теорией оценки эффективности систем, качество объекта, в том числе СЗИ, проявляется лишь в процессе его использования по назначению, поэтому наиболее объективным является оценивание по эффективности применения. Причем, чем более конкретно сформулирована цель защиты информации, детально уяснены имеющиеся для этого ресурсы и определен комплекс ограничений, тем в большей степени можно ожидать получение желаемого результата. В этой связи содержание целевого назначения системы на формализованном уровне приобретает многомерный, векторный, нечеткий, субъективный характер, что при синтезе системы защищенных мероприятий приводит к необходимости решать задачи с многокритериальными показателями. В результате достаточно сложно, а зачастую и невозможно, оценить качество мероприятий информационной безопасности, а, соответственно, и определить, чем один вариант защищенных мероприятий лучше другого.

По мнению авторов, для решения задачи оценки эффективности мероприятий информационной безопасности на защищенных экономических системах в указанных условиях целесообразно применять аппарата искусственных нейронных сетей (ИНС).

В нейросетевом подходе задача оценки сводится к восстановлению нелинейной функции по набору вариантов реализации защитных мероприятий и их возможных результатов, имеющей вид:

$$
y=f_{y}\left(x_{1}, x_{2}, \ldots . x_{n}\right)
$$

для которого связь <входы $\left(x_{i}\right)$-выход(y)> представлена в виде матрицы экспертных оценок [2].

Указанную матрицу экспертных оценок можно представить в виде нечеткой базы знаний:

$$
\text { ЕСЛИ }\left\lfloor\left(\mathrm{x}_{1}=\mathrm{a}_{1}^{\mathrm{j} 1}\right) \text { и: }\left(\mathrm{x}_{\mathrm{i}}=\mathrm{a}_{\mathrm{i}}^{\mathrm{j} 1}\right) \text { и: }\left(\mathrm{x}_{\mathrm{n}}=\mathrm{a}_{\mathrm{n}}^{\mathrm{j} 1}\right)\right\rfloor
$$

(с весовым коэффициентом $\mathrm{w}_{\mathrm{j} 1}$ ):

$$
\text { : или }\left[\left(\mathrm{x}_{1}=\mathrm{a}_{1}^{\mathrm{j} \mathrm{k}_{\mathrm{j}}}\right) \text { и: }\left(\mathrm{x}_{\mathrm{i}}=\mathrm{a}_{\mathrm{i}}^{\mathrm{j} \mathrm{k}_{\mathrm{j}}}\right) \text { и: }\left(\mathrm{x}_{\mathrm{n}}=\mathrm{a}_{\mathrm{n}}^{\mathrm{j} \mathrm{k}_{\mathrm{n}}}\right)\right\rfloor
$$

(с весовым коэффициентом $\mathrm{w}_{\mathrm{jk}}$ )

$$
\text { TO } \quad y=f_{j} \quad j=\overline{1, m} \text {. }
$$

где $\mathrm{a}_{\mathrm{j}}^{\mathrm{p}}$ - лингвистический терм, описывающий входной параметр $\mathrm{x}_{\mathrm{i}}$ в строке $\mathrm{p}=\mathrm{k}_{\mathrm{j}}$;

$\mathrm{k}_{\mathrm{j}}$ - число строк, соответствующих классу $\mathrm{d}_{\mathrm{j}}$ выходного параметра у;

$\mathrm{w}_{\mathrm{jp}}$ - значение функции принадлежности, описывающей степень уверенности эксперта относительно высказывания $\mathrm{p}=\mathrm{k}_{\mathrm{j}}$.

Классы $d_{j}, j=\overline{1, m}$, результат дискретизации диапазона $[\underline{y}, \bar{y}]$ выходного параметра на т поддиапазонов:

$$
\lfloor y, \bar{y}]=\left[\mathrm{y}, \mathrm{y}_{1}\right) \cup \ldots \cup\left[\mathrm{y}_{\mathrm{j}-1}, \mathrm{y}_{\mathrm{j}}\right) \cup \ldots \cup\left[\mathrm{y}_{\mathrm{m}-1}, \overline{\mathrm{y}}\right]
$$

Исходя из описания (2), функция (1) представляется в следующем виде:

$$
\begin{aligned}
& \mathrm{y}=\frac{\underline{\mathrm{y}} \mu^{\mathrm{d}_{1}}(\mathrm{y})+\mathrm{y}_{1} \mu^{\mathrm{d}_{2}}(\mathrm{y})+\ldots+\mathrm{y}_{\mathrm{m}-1} \mu^{\mathrm{d}_{\mathrm{m}}}(\mathrm{y})}{\mu^{\mathrm{d}_{1}}(\mathrm{y})+\mu^{\mathrm{d}_{2}}(\mathrm{y})+\ldots \mu^{\mathrm{d}_{\mathrm{m}}}(\mathrm{y})} \\
& \mu^{\mathrm{d}_{\mathrm{j}}}(\mathrm{y})=\max _{\mathrm{p}=1, \mathrm{k}_{j}}\left\{\mathrm{w}_{\mathrm{jp}} \min _{\mathrm{i}=1, \mathrm{n}}\left[\mu^{\mathrm{jp}}\left(\mathrm{x}_{\mathrm{i}}\right)\right]\right\} \\
& \mu^{\mathrm{jp}}\left(\mathrm{x}_{\mathrm{i}}\right)=\frac{1}{1-\left(\frac{\mathrm{x}_{\mathrm{i}}-\mathrm{b}_{\mathrm{i}}^{\mathrm{jp}}}{\mathrm{c}_{\mathrm{i}}^{\mathrm{jp}}}\right)^{2}}, \mathrm{i}=\overline{1, \mathrm{n}}, \mathrm{j}=\overline{1, \mathrm{n}}, \mathrm{p}=k_{j},
\end{aligned}
$$

где $\mu \mathrm{d}_{\mathrm{j}}(\mathrm{y})$ - функция принадлежности выходного параметра у к классу $\mathrm{d}_{\mathrm{j}} \in\left[\mathrm{y}_{\mathrm{j}-1}, \mathrm{y}_{\mathrm{j}}\right\rfloor$;

$\mu^{\mathrm{jp}}\left(\mathrm{x}_{\mathrm{i}}\right)-$ функция принадлежности входного параметра $\mathrm{x}_{\mathrm{i}}$ к терму $\mathrm{a}_{\mathrm{i}}^{\mathrm{p}}$;

$b_{i}^{j p}, c_{i}^{j p}-$ коэффициенты, корректирующие значения соответствующих функций принадлежности.

Представим лингвистическую информацию (1) в искусственной нейронной сети (2), структура которой изображена на рисунке 1 [3]. 


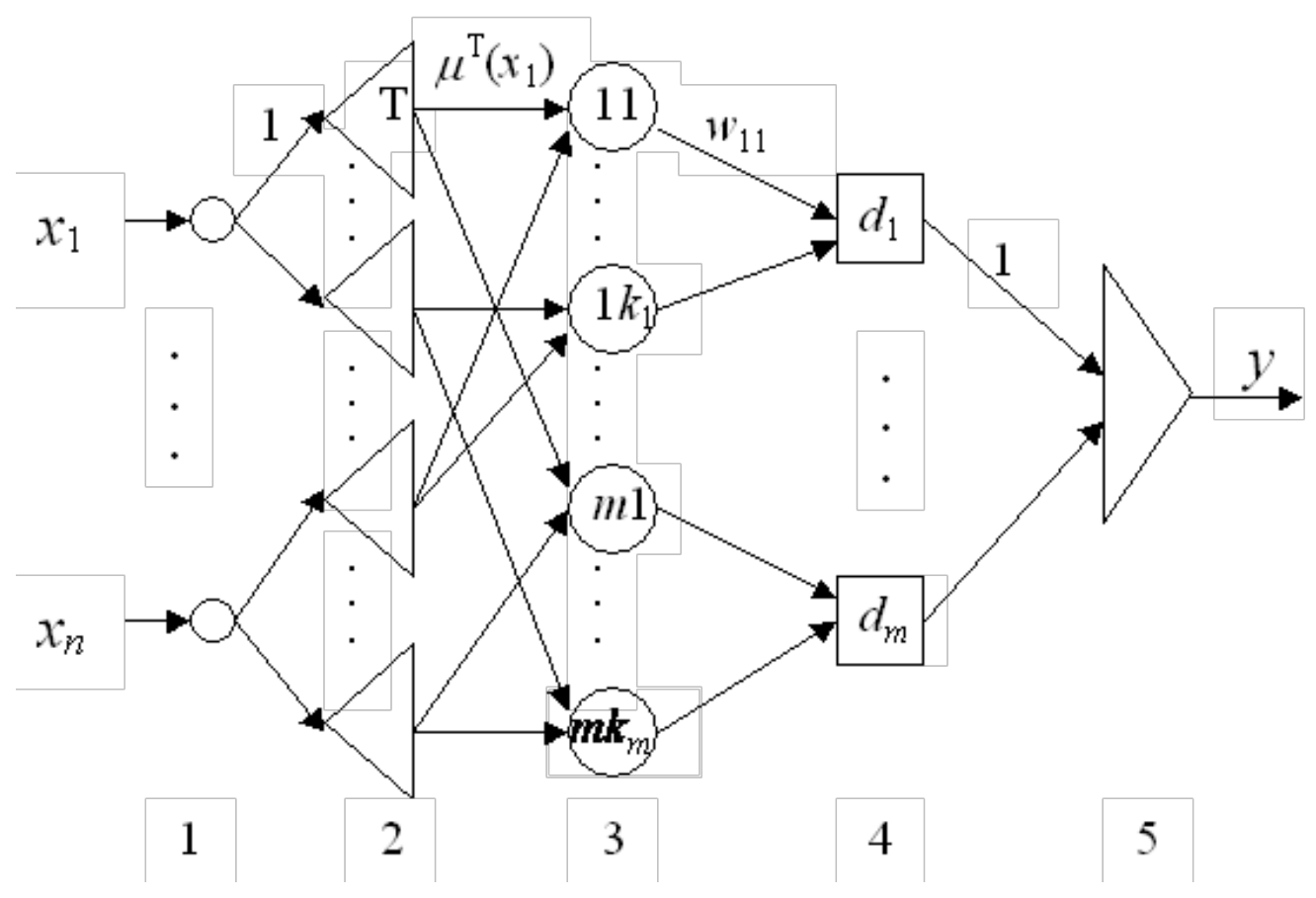

Puc. 1. Структура искусственной нейронной сети

где: $\mu^{\mathrm{T}}(\mathrm{u})-$ функция принадлежности переменной и к терму Т;

$\overline{\mathrm{dj}}-$ центр класса $\mathrm{d}_{\mathrm{j}} \in[\underline{\mathrm{y}}, \overline{\mathrm{y}}]$.

Представленная искусственная нейронная сеть пятислойная. Назначение слоев представлено ниже:

1 - входные параметры объекта;

2 - нечеткие термы, описывающие входные параметры;

3 - строки матрицы экспертных оценок (2);

4 - правила формирования $\mathrm{d}_{\mathrm{j}}, \mathrm{j}=\overline{1, \mathrm{~m}}$;

5 - операция преобразования нечёткого множества в чёткое число (4).

При этом послойное число узлов в ИНС определяется следующим образом:

в первом - по количеству входных параметров объекта анализа;

во втором - по количеству нечетких термов в матрице экспертных оценок (2);

в третьем - по количеству строк матрицы экспертных оценок;

в четвертом - по количеству классов выходного параметра.

При этом весовые коэффициенты дуг определяются следующим образом:

равным единице для дуг между 1-м и 2-м слоями;

равным значениям функциями принадлежности $\mu^{\mathrm{T}}(\mathrm{u})$ для дуг между 2-м и 3-м слоями;

равным весам правила формирования $\mathrm{d}_{j}$ для дуг между 3-м и 4-м слоями;

равным единице для дуг между 4-м и 5-м слоями.

Обучение нейронной сети осуществляется подбором весов минимизирующих невязку между оценкой и фактическим состоянием объекта анализа. Обучение ИНС осуществляется посредством реализации итерационной системы:

$$
\begin{aligned}
& w_{j p}(t+1)=w_{j p}(t)-\eta \frac{\partial E_{t}}{\partial w_{j p}(t)} \\
& c_{i}^{j p}(t+1)=c_{i}^{j p}(t)-\eta \frac{\partial E t}{\partial c_{i}^{j p}(t)} \\
& b_{i}^{j p}(t+1)=b_{i}^{j p}(t)-\eta \frac{\partial E t}{\partial b_{i}^{j p}(t)} \\
& j=\overline{1, m}, j=\overline{1, n}, p=k_{j},
\end{aligned}
$$


Критерием остановки итерационных вычислений является минимум функционала:

$$
\mathrm{E}_{\mathrm{t}}=\frac{1}{2}\left(\hat{\mathrm{y}}_{\mathrm{t}}-\mathrm{y}_{\mathrm{t}}\right)^{2}
$$

где:

$\hat{y}_{t}$ и $\mathrm{y}_{\mathrm{t}}-$ фактический и эмпирический выходные параметры объекта (1) на t-м шаге обучения;

$\mathrm{w}_{\mathrm{jp}}(\mathrm{t}), \mathrm{c}_{\mathrm{i}}^{\mathrm{jp}}(\mathrm{t}), \mathrm{b}_{\mathrm{i}}^{\mathrm{jp}}(\mathrm{t})$ - весовые коэффициенты $\mathrm{w}$ и параметры функций принадлежности $\mathrm{b}$ и с на t-м шаге обучения;

$\mathrm{m}$ - параметр обучения.

Частные производные, в (7)-(9), описывают уровень невязки параметров ИНС, и определяются следующими выражениями:

$$
\begin{aligned}
& \frac{\partial \mathrm{E}_{\mathrm{t}}}{\partial \mathrm{w}_{\mathrm{jp}}}=\varepsilon_{1} \varepsilon_{2} \varepsilon_{3} \frac{\partial \mu^{\mathrm{d}_{\mathrm{j}}}(\mathrm{y})}{\partial \mathrm{w}_{\mathrm{jp}}} \\
& \frac{\partial \mathrm{E}_{\mathrm{t}}}{\partial \mathrm{c}_{\mathrm{i}}^{\mathrm{jp}}}=\varepsilon_{1} \varepsilon_{2} \varepsilon_{3} \varepsilon_{4} \frac{\partial \mu^{\mathrm{jp}}\left(\mathrm{x}_{\mathrm{i}}\right)}{\partial \mathrm{c}_{\mathrm{i}}^{\mathrm{jp}}} \\
& \frac{\partial \mathrm{E}_{\mathrm{t}}}{\partial \mathrm{b}_{\mathrm{i}}^{\mathrm{jp}}}=\varepsilon_{1} \varepsilon_{2} \varepsilon_{3} \varepsilon_{4} \frac{\partial \mu^{\mathrm{jp}}\left(\mathrm{x}_{\mathrm{i}}\right)}{\partial \mathrm{b}_{\mathrm{i}}^{\mathrm{jp}}}
\end{aligned}
$$

где

$\varepsilon_{1}=\frac{\partial \mathrm{E}_{\mathrm{t}}}{\partial \mathrm{y}}=\mathrm{y}_{\mathrm{t}}-\hat{\mathrm{y}}_{\mathrm{t}}$

$\varepsilon_{2}=\frac{\partial y}{\mu^{d_{j}}(y)}=\frac{\bar{d} \sum_{j=1}^{m} \mu^{d_{j}}(y)-\sum_{j=1}^{m} d_{j} \mu^{d_{j}}(y)}{\left(\sum_{j=1}^{m} \mu^{d_{j}}(y)\right)^{2}}$

$$
\varepsilon_{3}=\frac{\delta \mu^{\mathrm{d}_{\mathrm{j}}}(\mathrm{y})}{\delta\left(\prod_{\mathrm{i}=1}^{\mathrm{n}} \mu^{\mathrm{jp}}\left(\mathrm{x}_{\mathrm{i}}\right)\right)}=\mathrm{w}_{\mathrm{jp}}
$$

$$
\varepsilon_{4}=\frac{\partial\left(\prod_{i=1}^{n} \mu^{j p}\left(x_{i}\right)\right)}{\partial \mu^{j p}\left(x_{i}\right)}=\frac{1}{\mu^{j p}\left(x_{i}\right)} \prod_{i=1}^{n} \mu^{j p}\left(x_{i}\right)
$$

$$
\frac{\mu^{\mathrm{d}_{\mathrm{j}}}(\mathrm{y})}{\delta \mathrm{w}^{\mathrm{jp}}\left(\mathrm{x}_{\mathrm{i}}\right)}=\prod_{\mathrm{i}=1}^{\mathrm{n}} \mu^{\mathrm{jp}}\left(\mathrm{x}_{\mathrm{i}}\right)
$$

$$
\begin{gathered}
\frac{\partial \mu^{j p}\left(x_{i}\right)}{\partial c_{i}^{j p}}=\frac{2 c_{i}^{j p}\left(x_{i}-b_{i}^{j p}\right)^{2}}{\left(\left(c_{i}^{j p}\right)^{2}\left(x_{i}-b_{i}^{j p}\right)^{2}\right)^{2}} \\
\frac{\partial \mu^{j p}\left(x_{i}\right)}{\partial b_{i}^{j p}}=\frac{2\left(c_{i}^{j p}\right)^{2}\left(x_{i}-b_{i}^{j p}\right)}{\left(\left(c_{i}^{j p}\right)^{2}\left(x_{i}-b_{i}^{j p}\right)^{2}\right)^{2}}
\end{gathered}
$$

Итак, алгоритм обучения ИНС реализуется в два этапа. На первом - осуществляется расчет значения выходного параметра (у), соответствующее архитектуре ИНС. На втором - значение невязки $\left(\mathrm{E}_{\mathrm{t}}\right)$ и по формулам (11)-(20) пересчитываются весовые коэффициенты связей межу нейронами.

Таким образом, предложенный подход к решению задачи оценки эффективности мероприятий информационной безопасности на защищенных экономических системах реализован в виде ИНС, объединяющей возможности обработки гетерогенной информации об оцениваемой СЗИ, представленной в виде количественных данных и сложных качественных лингвистических описаний, полученных на основе экспертных оценок, и обучения ИНС в реальном масштабе времени. При этом привлечение возможностей ИНС для получения адекватной модели, анализируемой СЗИ, позволяет существенно повысить объективность и точность оценивания и, как следствие, его качество. 


\section{Библиографический список}

1. ГОСТ Р 50922-96. Защита информации. Основные термины и определения.

2. Скляров А.В., Тищенко Е.Н., Стрюков М.Б., Шарыпова Т.Н. Управление информационными рисками защищенных экономических систем на основе анализа нечетких временных рядов// Вопросы экономики и права № 82016 с.58

3. отштейн А.П. Интеллектуальные технологии идентификации. Универсум. Винница 1999 